\title{
A study on interstitial heat transfer in consolidated and unconsolidated porous media
}

\author{
Akira Nakayama ${ }^{1,2}$, Kenji Ando ${ }^{3}$, Chen Yang ${ }^{1}$, Yoshihiko Sano ${ }^{1}$, Fujio Kuwahara ${ }^{1}$ and Jianjun Liu ${ }^{2}$ \\ ${ }^{1)}$ Department of Mechanical Engineering, Shizuoka University \\ 3-5-1 Johoku, Hamamatsu, 432-8561 Japan \\ ${ }^{2)}$ Department of Civil Engineering, Wuhan Polytechnic University \\ Hubei, Wuhan 430023, China \\ ${ }^{3)}$ Research Division, Nitto Kogyo Corporation \\ 2201, Kanihara, Nagakute-Cho, Aichi-Gun, aichi, 480-1189 Japan
}

Keywords: Interstitial heat transfer, Porous media, Packed bed, Cellular ceramics, Foam materials

\begin{abstract}
The Nusselt number expressions are presented for the interstitial heat transfer coefficients for both consolidated and unconsolidated porous media. A boundary layer consideration is made for unconsolidated porous media to derive a general Nusselt number correlation, which shows its square root dependence of the Reynolds number, and matches fairly well with existing experimental data and correlations. As for low density consolidated porous media, a rigorous mathematical arguments based on the volume averaging theory are provided, so as to explain the reason why the Reynolds number exponent of the Nusselt number expression for the case of low density consolidated porous media is much greater than that of unconsolidated porous media. The resulting expressions are compared against available experimental data and empirical correlations, and found to be in good accord with them.
\end{abstract}




\section{INTRODUCTION}

A number of empirical correlations for interstitial heat transfer coefficients have been proposed for unconsolidated porous media. Wakao and Kaguei [1] give the history of the development in determination of the interstitial heat transfer coefficient for the case of unconsolidated porous media. Upon assembling available experimental data whose Reynolds numbers based on the particle diameter are greater than 10 , they eventually proposed a general empirical correlation for the packed beds, in which the Reynolds number exponent for the Nusselt number is 0.6, while its Prandtl number exponent is $1 / 3$. Their correlation has been frequently used in many engineering applications associated with packed beds comprised of spherical particles. Kim and Jang [2] provide extensive discussion on the interstitial heat transfer in porous media and a general criterion for the local thermal equilibrium. Recent state of art reviews can also be found elsewhere [3, 4].

Heat transfer characteristics associated with consolidated materials, such as sintered metals, cellular ceramics and ceramic foams and many other foam materials, have also gained extensive research attention because of possible engineering applications such as solid matrix heat exchangers [5], electronic cooling [6] and structural components in gas turbines [7]. Fu et al. [7] carried out exhaustive measurements and correlated the interstitial heat transfer coefficients of cellular materials, which indicates the linear dependency of the Reynolds number on the Nusselt number. They found that the volumetric heat transfer coefficient increases with a decrease in specimen thickness to the mean pore diameter ratio. Maiorov et al. [8] collected heat transfer data using sintered metal and found that the exponent ranges from 0.9 to 1.34. while Kar and Dybbs [9] found their data follow their correlation well with the exponent being 1.35. A general correlation for the volumetric heat transfer coefficient was introduced by Kamiuto and Yee [10] for open cellular porous materials, who indicated the Reynolds number exponent being close to 0.8. They assembled experimetal data reported by Younis and Viskanta [11], Ichimiya [12] and themselves, to find that all these experimental data for the volumetric heat transfer coefficient can be correlated fairly well using the strut diameter as a chracteristic length for the open cellular materials.

It is interesting to note that the Reynolds number exponent for the case of low density consolidated porous media (i.e. high porosity media) is around 1, much higher than 0.5 to 0.6 for the case of unconsolidated porous media. Since the exponents for convective heat transfer in internal channels are 1/3 and 0.8 for laminar flow and turbulent flow, respectively, somewhat different convective heat transfer mechanism should take place within low density consolidated porous media to exhibit the exponent as large as 1 . No theoretical explanations to this difference in the Reynolds number dependency experimentally observed between consolidated and unconsolidated media seem to have reported in the literature. 
In this paper, firstly, we shall investigate the interstitial heat transfer within unconsolidated porous media such as packed beds of spherical particles. The boundary layer consideration is made to derive a general expression for the interstitial heat transfer coefficient. Then, we shall focus on the interstitial heat transfer in the low density consolidated porous media. In order to ellucidate a unique heat transfer mechanism (leading to the linear Reynolds number dependence on the Nusselt number), we shall appeal to a rigorous mathematical modelling based on the volume averaging theory and reduce a general expression appropriate for the volumetric heat transfer coefficient within low density consolidated porous media.

\section{BOUNDARY LAYER CONSIDERATION FOR UNCONSOLIDATED POROUS MEDIA}

An unconsolidated porous medium is schematically shown in Figure 1, in which spherical particles are placed uniformly in a three-dimensional space. The fluid passes through the particles whose surface temperature may be higher or lower than that of the passing fluid. Since both velocity and temperature fields become fully developed downstream beyond a few rows, we may assume that the heat and fluid flow in consideration is steady and periodically fully developed.

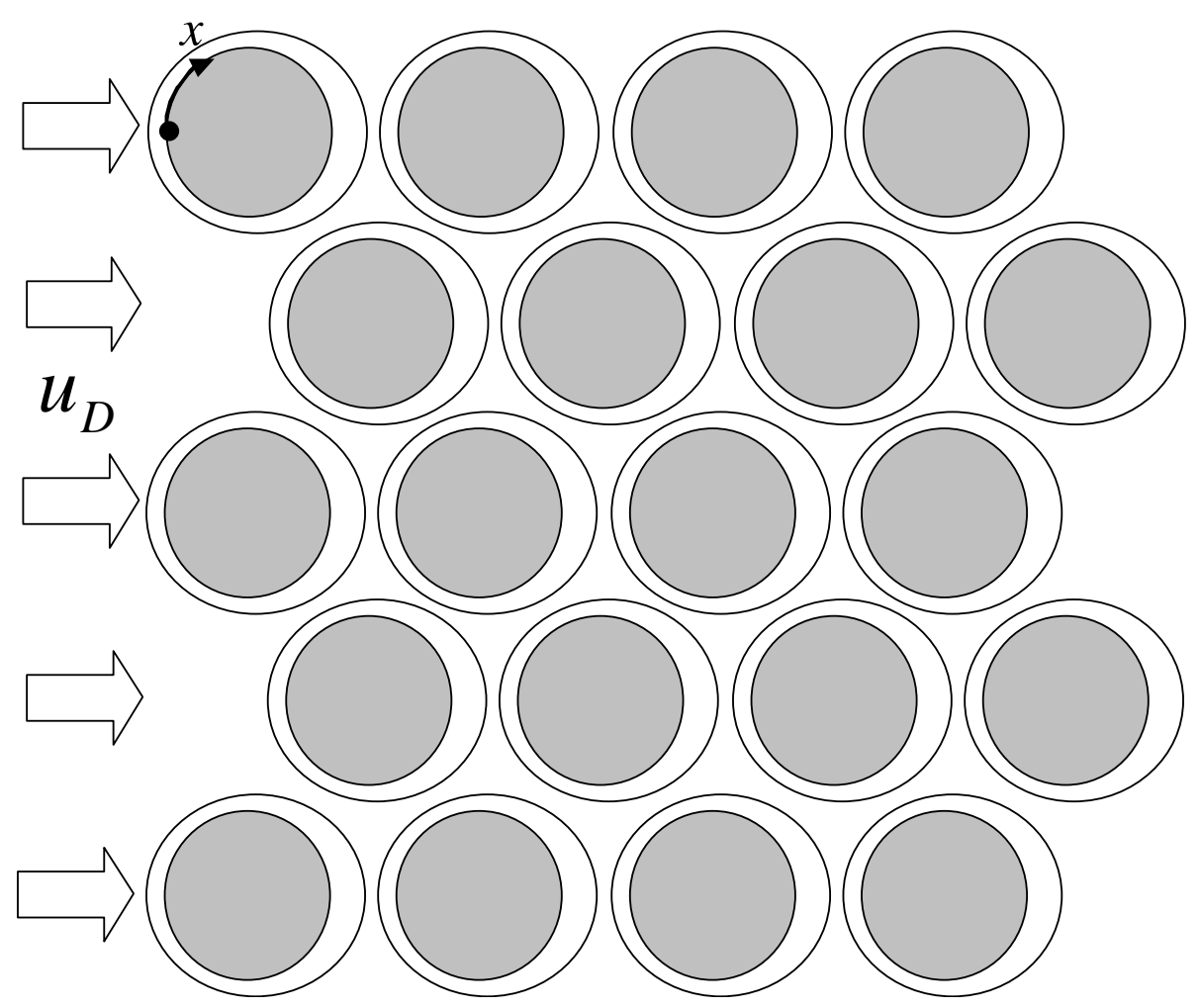

Figure 1 Unconsolidated porous medium 
The bulk mean temperature of the fluid varies spatially as the fluid passes through the particles, since heat transfer takes place between the particles and fluid. However, the local temperature field around each particle stays similar for periodically fully developed heat and fluid flows. As the figure indicates, the thermal boundary layer formed over the leeward surface of a particle must share the space with the thermal boundary layer formed at the windward surface of the particle behind it. Therefore, it is quite natural to assume that the thermal boundary layer thickness remains nearly constant over an entire wall surface of the particle, as illustrated in the figure.

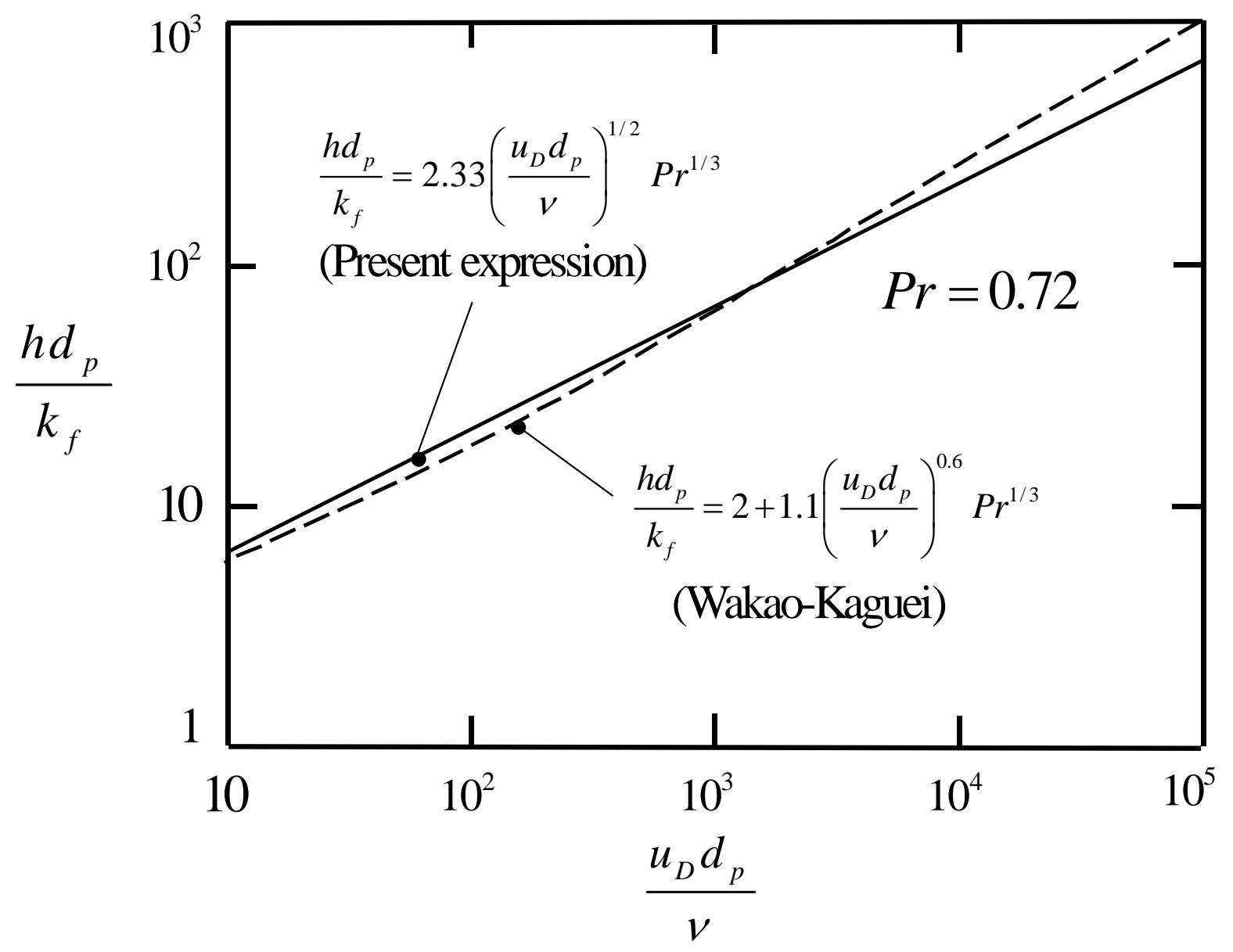

Figure 2 Comparison of the present formula with the empirical correlation for packed beds

The thermal boundary layer thickness around the particle may be estimated assuming an axisymmetric stagnation flow. The local heat transfer coefficient $h(x)$ based on the asymptotic solution e.g. [14] for the Falkner-Skan forced convection, where the external velocity follows the relation $u_{e}(x) \propto x^{m}$, is given by 
$\frac{h x}{k_{f}}=0.489 \sqrt{i}\left(\left(1+\frac{m}{i}\right) f^{\prime \prime}\left(0 ; \frac{m}{i}\right)\right)^{1 / 3}\left(\frac{u_{e} X}{v}\right)^{1 / 2} \operatorname{Pr}^{1 / 3}$

where $x$ is the distance measured from the front stagnation point as indicated in Figure 1, while the integar $i$ takes the value 1 for the plane flows and 3 for the axisymmetric flows. Furthermore, $f^{\prime \prime}\left(0 ; \frac{m}{i}\right)=\left(\frac{\tau_{w}}{\rho u_{e}^{2}}\right)\left(\frac{u_{e} x}{v}\right)^{1 / 2}$ is the dimensionless wall friction which can readily be obtained from the Falkner-Skan equation. For the stagnation flow over a sphere, we have $m=1, i=3$, $f^{\prime \prime}(0 ; 1 / 3)=0.757$ and $u_{e}(x)=3\left(u_{D} / \varepsilon\right)\left(x / d_{p}\right)$, where $\varepsilon$ is the porosity and $u_{D}$ is the Darcian velocity. Hence, Equation (1) reduces to

$\frac{h d_{p}}{k_{f}}=1.47\left(\frac{u_{D} d_{p}}{\varepsilon v}\right)^{1 / 2} \operatorname{Pr}^{1 / 3}=2.33\left(\frac{u_{D} d_{p}}{v}\right)^{1 / 2} \operatorname{Pr}^{1 / 3}: \varepsilon=0.4$

In Figure 2, the foregoing equation is plotted along with the empirical correlation established by Wakao and Kaguei [1] for closely packed beds ( $\varepsilon=0.4$ ):

$\frac{h d_{p}}{k_{f}}=2+1.1\left(\frac{u_{D} d_{p}}{v}\right)^{0.6} \operatorname{Pr}^{1 / 3} \quad$ : Wakao and Kaguei (Empirical)

Despite slight difference in the exponents, both correlations agree well over a wide range of the Reynolds number.

\section{VOLUME AVERAGING CONSIDERATION FOR CONSOLIDATED POROUS MEDIA}

A typical structure of a consolidated porous medium is shown in Figure 3. In low density consolidated porous media, the fluid particles find their way in the voids, which are connected and aligned in the direction of the macroscopic flow (i.e. the direction of the macroscopic pressure gradient). As illustrated in Figure 4, each of these (imaginary) primary passages is surrounded by numerous (imaginary) secondary passages, which are connected to the primary passage at various inclination angles. As the fluid particles pass through the primary passage to form a major current, some fluid (in local thermal equilibrium with the solid matrix) flows from its secondary passage to the primary passage, while the same amount of the fluid (in local thermal equilibrium with the major current) flows back from the primary passage to the secondary passage, so as to satisfy the continuity principle. Since the solid matrix is continuous in a consolidated medium (unlike in a unconsolidated medium), the fluid hid within the secondary passage is likely to be in local thermal equilibrium with the solid structure of the secondary passage. This enthalpy exchange associated with the secondary passages is responsible for the interstitial heat transfer within a low density consolidated porous medium. We shall explain this peculiar heat transfer mechanism, using a 
simplified mathematical model as depicted in Figure 4. In the figure, the primary passages are illustrated as a series of connected voids, while the secondary passages together with the solid matrix structure are modeled collectively as a continuous porous medium saturated with a fluid. A similar double porosity treatment may be found in the study of fractured porous media associated with oil reservoirs [13].

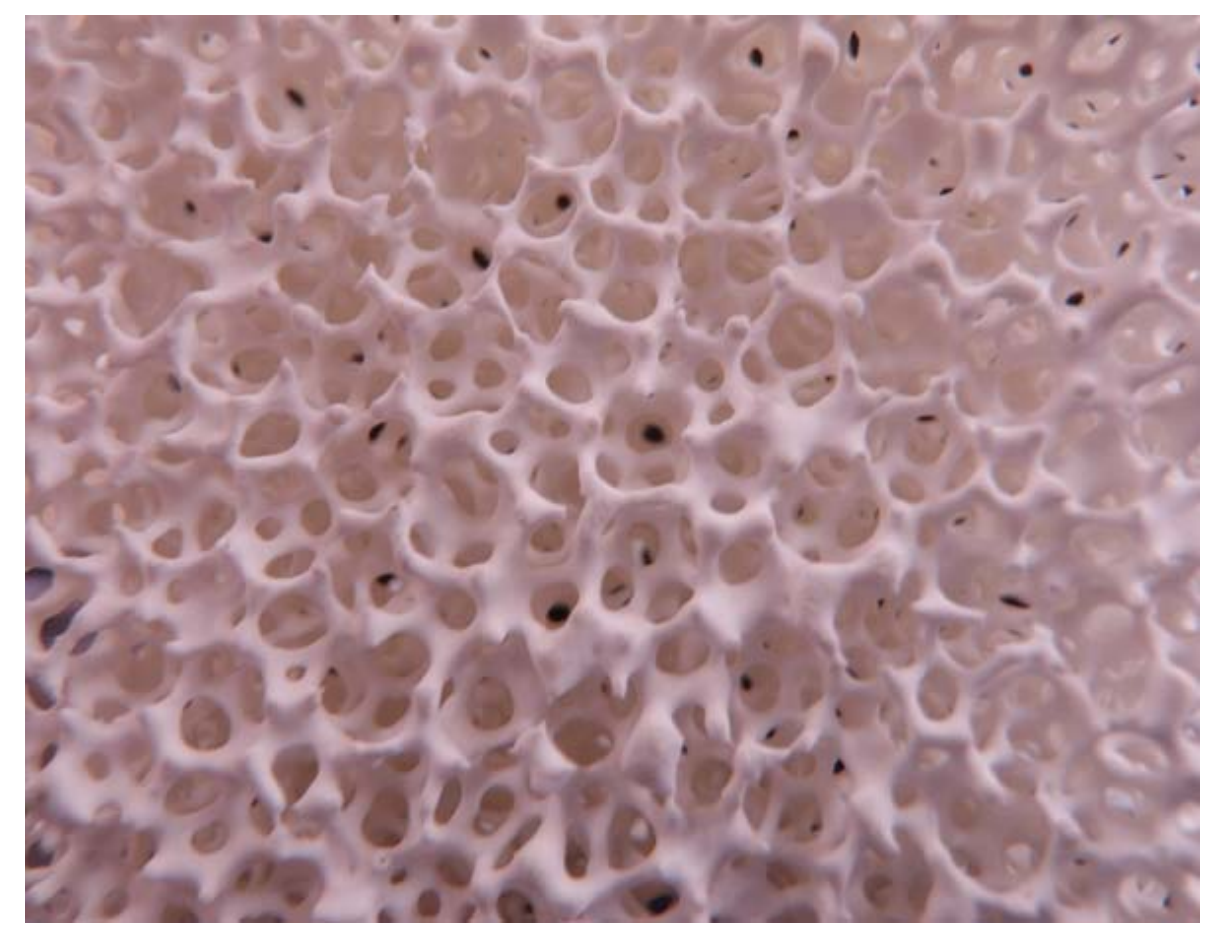

Figure 3 Consolidated porous medium

Thus, we shall consider the energy equation for the fluid phase in the primary passages and that for the solid matrix phase (saturated with a fluid), corresponding to the continuous solid structure forming secondary passages.

For the fluid phase:

$\rho_{f} c_{p_{f}} \frac{\partial}{\partial x_{j}} u_{j} T=\frac{\partial}{\partial x_{j}}\left(k_{f} \frac{\partial T}{\partial x_{j}}\right)$

For the matrix phase:

$\rho_{f} c_{p_{f}} \frac{\partial}{\partial x_{j}} u_{j} T=\frac{\partial}{\partial x_{j}}\left(k_{s} \frac{\partial T}{\partial x_{j}}\right)$

where the subscripts $f$ and $s$ stand for the fluid phase and matrix phase, respectively. Also note that $u_{j}$ in Equation (5) is the apparent velocity whereas $k_{s}$ is the effective thermal conductivity of the fluid saturated matrix. 


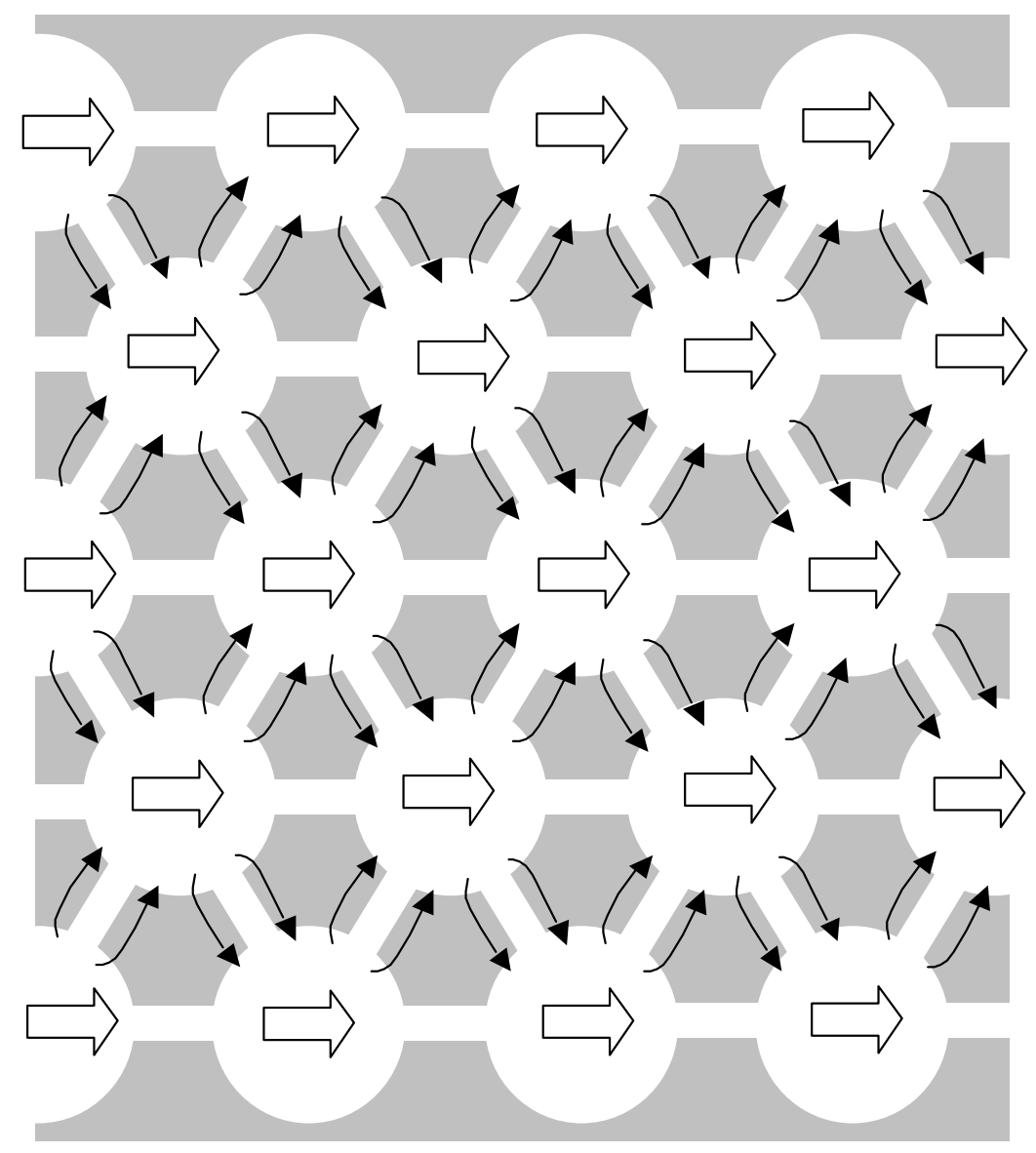

Figure 4 Simplified model for a consolidated porous medium

We consider a control volume $V$ in a fluid-saturated porous medium, whose length scale $V^{1 / 3}$ is much smaller than the macroscopic characteristic length, but, at the same time, much greater than the structural characteristic length (see e.g. Nakayama [14]). Under this condition, the volume average of a certain variable $f$ is defined as

$\langle\phi\rangle^{f} \equiv \frac{1}{V_{f}} \int_{V_{f}} \phi d V$

where $V_{f}$ is the volume space which the fluid phase (i.e. the primary passages) occupies. The porosity $\varepsilon \equiv V_{f} / V$ is the volume fraction of the fluid space. Following Nakayama [14], Cheng [15], Quintard and Whitaker [16] and many others, we decompose a variable into its intrinsic average and the spatial deviation from it:

$\phi=\langle\phi\rangle^{f}+\tilde{\phi}$ 
We shall exploit the following spatial average relationships:

$$
\begin{aligned}
& \left\langle\phi_{1} \phi_{2}\right\rangle^{f}=\left\langle\phi_{1}\right\rangle^{f}\left\langle\phi_{2}\right\rangle^{f}+\left\langle\tilde{\phi} \tilde{\phi}_{2}\right\rangle^{f} \\
& \left\langle\frac{\partial \phi}{\partial x_{i}}\right\rangle^{f}=\frac{1}{\varepsilon} \frac{\partial \varepsilon\langle\phi\rangle^{f}}{\partial x_{i}}+\frac{1}{V_{f}} \int_{A_{\mathrm{nt}}} \phi n_{i} d A
\end{aligned}
$$

where $A_{\mathrm{nnt}}$ is the local interfacial area between the blood and solid matrix, while $n_{i}$ is the unit vector pointing outward from the fluid side to solid side. Upon noting that the similar relationships hold also for the matrix phase, we obtain the volume averaged set of the energy equations as follows:

For the fluid phase:

$$
\begin{aligned}
& \rho_{f} c_{p_{f}} \varepsilon \frac{\partial}{\partial x_{j}}\left\langle u_{j}\right\rangle^{f}\langle T\rangle^{f} \\
& =\frac{\partial}{\partial x_{j}}\left(\varepsilon k_{f} \frac{\partial\langle T\rangle^{f}}{\partial x_{j}}+\frac{k_{f}}{V} \int_{A_{\mathrm{tu}}} T n_{j} d A-\varepsilon \rho_{f} c_{p_{f}}\left\langle\tilde{u}_{j} \tilde{T}\right\rangle^{f}\right)+\frac{1}{V} \int_{A_{\mathrm{tr}}} k_{f} \frac{\partial T}{\partial x_{j}} n_{j} d A-\frac{1}{V}\left(\int_{A_{\mathrm{tut}}} \rho_{f} c_{p_{f}} u_{j} T\right) n_{j} d A
\end{aligned}
$$

For the solid matrix phase:

$$
\begin{aligned}
& \rho_{f} c_{p_{f}}(1-\varepsilon) \frac{\partial}{\partial x_{j}}\left\langle u_{j}\right\rangle^{s}\langle T\rangle^{s} \\
& =\frac{\partial}{\partial x_{j}}\left((1-\varepsilon) k_{s} \frac{\partial\langle T\rangle^{s}}{\partial x_{j}}+\frac{k_{s}}{V} \int_{A_{\mathrm{m} t}} T n_{j} d A-(1-\varepsilon) \rho_{f} c_{p_{f}}\left\langle u_{j} \tilde{T}\right\rangle^{s}\right)-\frac{1}{V} \int_{A_{\mathrm{Ant}}} k_{f} \frac{\partial T}{\partial x_{j}} n_{j} d A+\frac{1}{V}\left(\int_{A_{\mathrm{mit}}} \rho_{f} c_{p_{f}} u_{j} T\right) n_{j} d A
\end{aligned}
$$

where $\langle T\rangle^{f}$ is the intrinsic average of the fluid temperature, while $\langle T\rangle^{s}$ is the intrinsic average of the matrix temperature. The second term on the right hand-side of Equation (10) describes the conductive heat transfer on the interface between the primary passage and the matrix. It is the last term on the right hand-side of Equation (10) that accounts for the effects of the back and forth seepage flows (through the secondary passages) on the interstitial heat transfer. Obviously, the first term on the right hand-side of Equation (10) denotes the diffusive heat transfer, which may well be neglected since the convection dominates over the diffusion within the primary passages. As illustrated in the simplified model shown in Figure 4, the fluid, which seeps from the matrix into the void, flows back from the void into the matrix. In such back and forth fluid motions, the volume averaged velocity $\left\langle u_{j}\right\rangle^{s}$ in the matrix remains virtually zero (i.e. $u_{j}=\tilde{u}_{j}$ ) such that the convection term (i.e. the left hand side term) in the matrix energy equation (11), vanishes. Consequently, these volume averaged energy equations reduce to 
$\rho_{f} c_{p_{f}} \varepsilon \frac{\partial}{\partial x_{j}}\left\langle u_{j}\right\rangle^{f}\langle T\rangle^{f}=\frac{1}{V} \int_{A_{\text {Ant }}} k_{f} \frac{\partial T}{\partial x_{j}} n_{j} d A-\frac{1}{V}\left(\int_{A_{\text {Ant }}} \rho_{f} c_{p_{f}} u_{j} T\right) n_{j} d A$

and

$\frac{\partial}{\partial x_{j}}\left((1-\varepsilon) k_{s} \frac{\partial\langle T\rangle^{s}}{\partial x_{j}}+\frac{k_{s}}{V} \int_{A_{\mathrm{tat}}} T n_{j} d A-(1-\varepsilon) \rho_{f} c_{p_{f}}\left\langle u_{j} \tilde{T}\right\rangle^{s}\right)=\frac{1}{V} \int_{A_{\mathrm{tut}}} k_{f} \frac{\partial T}{\partial x_{j}} n_{j} d A-\frac{1}{V}\left(\int_{A_{\mathrm{tat}}} \rho_{f} c_{p_{f}} u_{j} T\right) n_{j} d A$

which may be combined to give

$\rho_{f} c_{p_{f}} \varepsilon \frac{\partial}{\partial x_{j}}\left\langle u_{j}\right\rangle^{f}\langle T\rangle^{f}=\frac{1}{V} \int_{A_{\text {mat }}} k_{f} \frac{\partial T}{\partial x_{j}} n_{j} d A-\frac{1}{V}\left(\int_{A_{\text {mat }}} \rho_{f} c_{p_{f}} u_{j} T\right) n_{j} d A=\frac{\partial}{\partial x_{j}}\left(k_{e f f} \frac{\partial\langle T\rangle^{s}}{\partial x_{j}}\right)$

where the divergence terms in the matrix phase are modeled using the gradient hypothesis as

$(1-\varepsilon) k_{s} \frac{\partial\langle T\rangle^{s}}{\partial x_{j}}+\frac{k_{s}}{V} \int_{A_{\text {trt }}} T n_{j} d A-(1-\varepsilon) \rho_{f} c_{p_{f}}\left\langle u_{j} \tilde{T}\right\rangle^{s}=k_{\text {eff }} \frac{\partial\langle T\rangle^{s}}{\partial x_{j}}$

The foregoing equation reveals that the mechanical dispersion $-(1-\varepsilon) \rho_{f} c_{p_{f}}\left\langle u_{j} \tilde{T}\right\rangle^{s}$ resulting from the back and forth fluid motions within the secondary passages of the matrix is fully responsible for the increase in the effective thermal conductivity of the matrix, hence, for the enhancement of the interstitial heat transfer.

\section{HEAT TRANSFER MODELING FOR CONSOLIDATED POROUS MEDIA}

The first right hand-side term in Equation (12), associated with the surface integral, describes the conductive heat transfer on the interface between the primary passage and the matrix. The term may be modeled using Newton's cooling law as

$\frac{1}{V} \int_{A_{\mathrm{ntu}}} k_{f} \frac{\partial T}{\partial x_{j}} n_{j} d A=a_{f} h\left(\langle T\rangle^{s}-\langle T\rangle^{f}\right)$

where $a_{f}$ and $h$ are the specific surface area and interfacial heat transfer coefficient, respectively.

As for modeling the second term on the right hand-side of Equation (12), describing the interstitial heat transfer due to the back and forth seepage flows (through the secondary passages), we note that the fluid in the secondary passage is in local thermal equilibrium with the consolidated solid matrix. Thus, this fluid in the secondary passage at the temperature $\langle T\rangle^{s}$ flows into the primary passage and then flows back into the secondary passage at the temperature $\langle T\rangle^{f}$, as 
illustrated in Figure 5. Obviously, this seepage flow results in the net heat transfer from the matrix phase to the fluid phase, which can be modeled as

$-\frac{1}{V}\left(\int_{A_{\text {int }}} \rho_{f} c_{p_{f}} u_{j} T\right) n_{j} d A=\rho_{f} c_{p_{f}} u_{\text {int }} a_{f}\left(\langle T\rangle^{s}-\langle T\rangle^{f}\right)$

where $u_{\text {int }}$ is the seepage velocity at the interface.

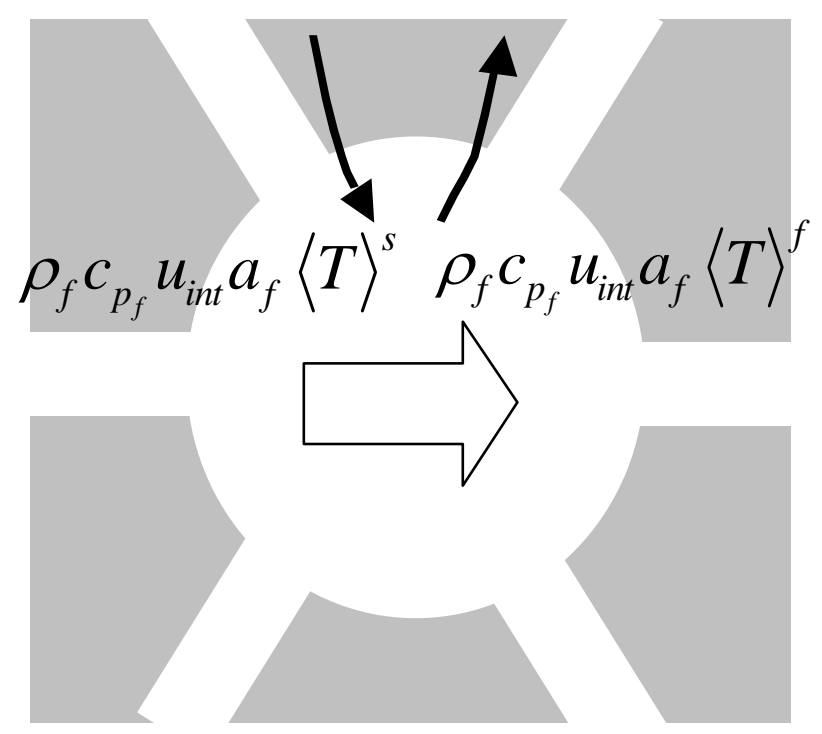

Figure 5 Net heat transfer due to seepage flow

Upon having modeled the surface integral terms associated with the conductive interfacial heat transfer and the seepage convective heat transfer, we may rewrite the energy equation (12) for the fluid phase as

$$
\rho_{f} c_{p_{f}} \varepsilon \frac{\partial}{\partial x_{j}}\left\langle u_{j}\right\rangle^{f}\langle T\rangle^{f}=a_{f}\left(h+\rho c_{p_{f}} u_{\text {int }}\right)\left(\langle T\rangle^{s}-\langle T\rangle^{f}\right)
$$

The foregoing equation reveals that what we can measure is not the heat transfer coefficient $h$ itself, but the effective heat transfer coefficient, namely, $h_{\text {eff }}=h+\rho c_{p_{t}} u_{\text {int }}$. Thus, the Nusselt number correlations reduced from most experimental data are actually based on the effective heat transfer coefficient: $N u=\frac{h_{\text {eff }} L_{\text {ref }}}{k_{f}}=\frac{\left(h+\rho c_{p_{f}} u_{\text {int }}\right) L_{\text {ref }}}{k_{f}}$ 
where $L_{\text {ref }}$ is a reference length. In the case of unconsolidated porous media, the seepage velocity $u_{\text {int }}$ is zero, hence, $N u=h L_{\text {ref }} / k_{f}$. Therefore, the boundary layer consideration is applicable as already demonstrated. However, in the case of low density consolidated porous media, we expect significant contribution from the seepage flow to the interstitial heat transfer such that

$$
N u=\frac{h_{\text {eff }} L_{\text {ref }}}{k_{f}}=c_{1}\left(\frac{u_{r e f} L_{r e f}}{v}\right)^{1 / 2} \operatorname{Pr}^{1 / 3}+\frac{\rho c_{p_{f}} u_{\text {int }} L_{\text {ref }}}{k_{f}} \cong \frac{\rho c_{p_{f}} u_{\text {int }} L_{\text {ref }}}{k_{f}}
$$

or

$$
N u_{v}=\frac{h_{v} L_{r e f}^{2}}{k_{f}} \cong \frac{\rho c_{p_{f}} u_{i n t} a_{f} L_{r e f}^{2}}{k_{f}}
$$

where $h_{v}=a_{f} h_{\text {eff }}$ is the volumetric heat transfer coefficient. The seepage flow velocity $u_{\text {int }}$ is likely to be proportional to the Darcian velocity $u_{D}$. Therefore, we may assume the Nusselt number based on the mean pore diameter $d_{m} \propto 1 / a_{f}$ to be in the form of

$$
N u_{v}=\frac{h_{v} d_{m}^{2}}{k_{f}}=c_{2} \frac{\rho c_{p_{f}} u_{D} d_{m}}{k_{f}}=c_{2}\left(\frac{u_{D} d_{m}}{v}\right) \operatorname{Pr}
$$

\section{COMPARISON OF THE PRESENT CORRELATION WITH AVAILABLE DATA}

As Equation (14) suggests that the proportional constant $c_{2}$ depends on the matrix structure, thermal conductivity and particular fluid-solid combination. Our exhaustive review on available experimental data on low density consolidated porous media, however, has revealed that $c_{2}$ is sensitive to the porosity, but is fairly insensitive to the others. In fact, the following correlation serves to cover a wide range of available experimental data:

$$
\begin{aligned}
N u_{v}=\frac{h_{v} d_{m}^{2}}{k_{f}}=0.07\left(\frac{\varepsilon}{1-\varepsilon}\right)^{2 / 3} & \left(\frac{u_{D} d_{m}}{v}\right) \operatorname{Pr} \\
: & \text { Present expression } \quad\left(0.7<\varepsilon<0.95,3<u_{D} d_{m} / v<1000\right)
\end{aligned}
$$

Fu et al. [7] appealed to the single blow experimental technique in conjunction with an inverse method, to determine the volumetric heat transfer coefficients in cellular ceramics. They used the least squares method to correlate their experimental data obtained for airflows ( $\operatorname{Pr}=0.72$ ). The correlations for mullite and cordierite ceramics specimens are given as follows:

$$
N u_{v}=0.275\left(\frac{u_{D} d_{m}}{v}\right)^{1.01} \quad \text { : Mullite }(\varepsilon=0.916)
$$




$$
N u_{v}=0.099\left(\frac{u_{D} d_{m}}{v}\right) \quad \text { : Cordierite }(\varepsilon=0.742)
$$

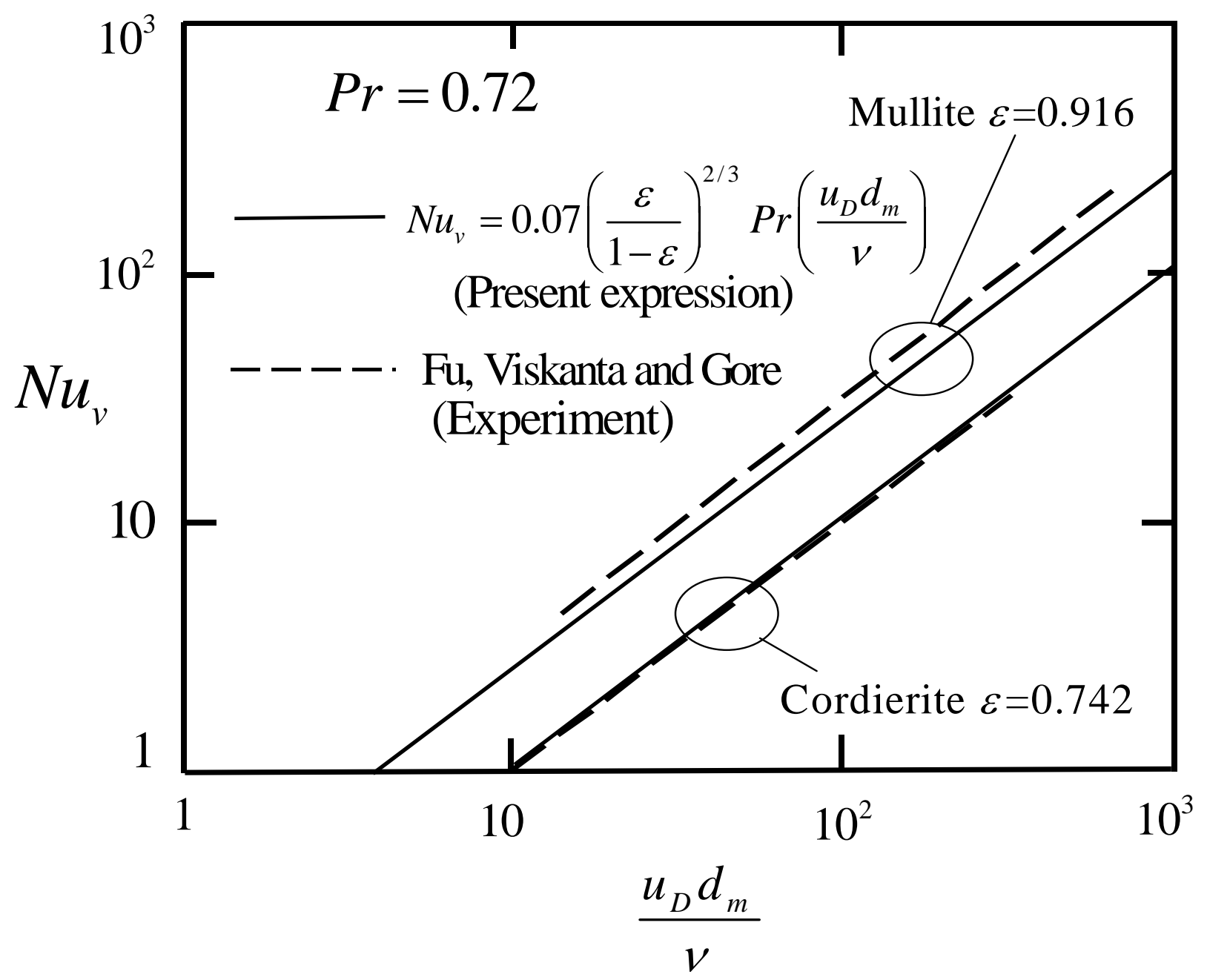

Figure 6 Comparison of the present expression with the experimental data of Fu et al.

These two empirical correlations are compared against the two lines generated from the present expression, namely, Equation (23), in Figure 6, where fairly good agreement can be confirmed.

Kamiuto and Yee [10] surveyed existing experimental data. Some data were obtained using the unsteady method (i.e. the single-blow methods), while the others were obtained from the steady methods. They carefully studied the collection of available experimental data on the volume heat transfer coefficients, and proposed a general correlation for the Nusselt number based on the equivalent strut diameter. Their correlation based on the equivalent strut diameter can be rewritten in favor of the mean pore diameter as follows: 
$N u_{v}=\frac{h_{v} d_{m}{ }^{2}}{k_{f}}=0.124\left(\frac{3 \pi \varepsilon}{4(1-\varepsilon)}\right)^{0.605}\left(\frac{u_{D} d_{m}}{v} \operatorname{Pr}\right)^{0.791} \quad$ : Kamiuto and Yee (Empirical)

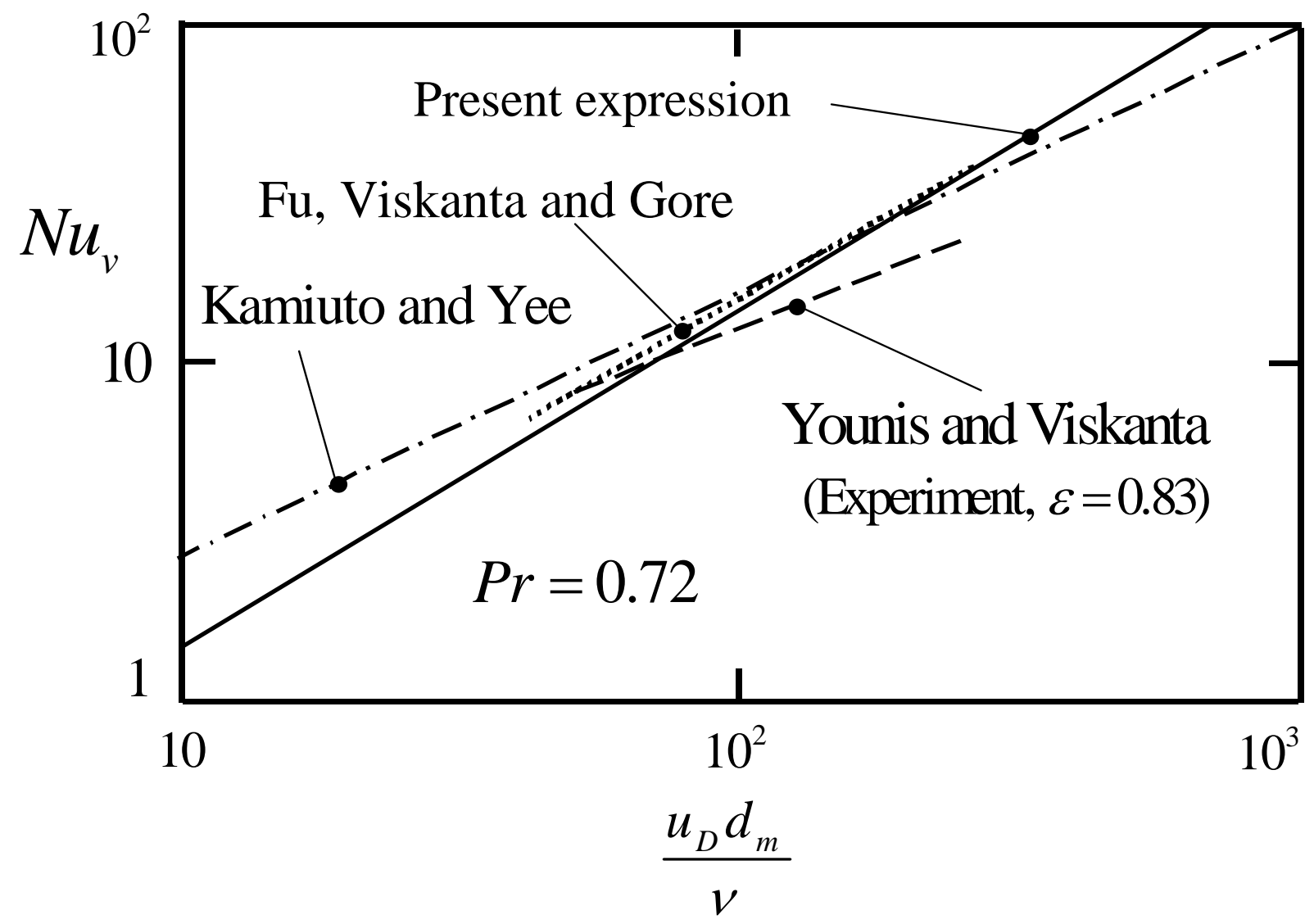

Figure 7 Comparison of various correlations with the experimental data of Younis and Viskanta

Their correlation reproduces most of the experimental data that they surveyed within an error of less than $40 \%$. Despite the difference in the exponents, the similarity observed between this general empirical correlation (25) and the present expression (23) is quite interesting. Younis and Viskanta [11] reported the volumetric heat transfer coefficient for the case of alumina open cellular materials. In figure 7, the experimental correlation obtained by them for $\varepsilon=0.83$ is plotted along with three formulas, namely, Kamiuto and Yee’s correlation, Fu, Viskanta and Gore's correlation and the present expression. All three correlations give the levels of the Nusselt number, reasonably close to that of the experimental data. However, the present expression and the correlation proposed by Fu et al., which are close to each other, give a slope higher than that indicated by the experiment for this case. Fu at al. claim that the discrepancy observed here is due to the differences in the pore geometry and thickness of the materials. 
Careful examination of the experimental data and correlations reveals that the present expression (23) is quite general and useful for estimating the volumetric heat transfer coefficients of low density consolidated porous media. The expression may serve as a reliable correlation of volumetric heat transfer coefficient for a wide range of the porosity, $0.7<\varepsilon<0.95$, and the Reynolds number based on the mean pore diameter, $3<u_{D} d_{m} / v<1000$.

\section{CONCLUSIONS}

The interstitial heat transfer coefficients in both consolidated and unconsolidated porous media were investigated in depth. A general set of heat transfer correlations were proposed and compared with existing experimental data and empirical correlations. Firstly, a boundary layer consideration was introduced to consider heat transfer in unconsolidated porous media. A general Nusselt number correlation was derived using the boundary layer solution for the axisymmetric stagnation thermal boundary layer, which shows its square root dependence of the Reynolds number, and matches fairly well with existing experimental data and correlations. Then, for low density consolidated porous media, a volume averaging theory was exploited to obtain the set of macroscopic energy equations. The energy equations along with mathematical modeling reveal the reason why the Reynolds number exponent of the Nusselt number expression for the case of low density consolidated porous media is much greater than that of unconsolidated porous media. The present expressions are compared against available experimental data and empirical correlations, and found to be valid for a wide range of the porosity and Reynolds number. The heat transfer mechanism associated with consolidated porous media can be investigated further using three-dimensional numerical simulations using periodic consolidated structures. Such a numerical attempt is underway.

\section{ACKNOWLEDGEMENTS}

The authors have benefited much from the remarks made by Prof. Sung Jin Kim of KAIST to the senior author during the $7^{\text {th }}$ JSME-KSME thermal and Fluids Engineering Conference held in Sapporo. This paper is dedicated to the memory of Prof. Kamiuto who deceased on September 2008 in recognition of his accomplishment in heat transfer in open cellular porous media. 


\section{NOMENCLATURE}

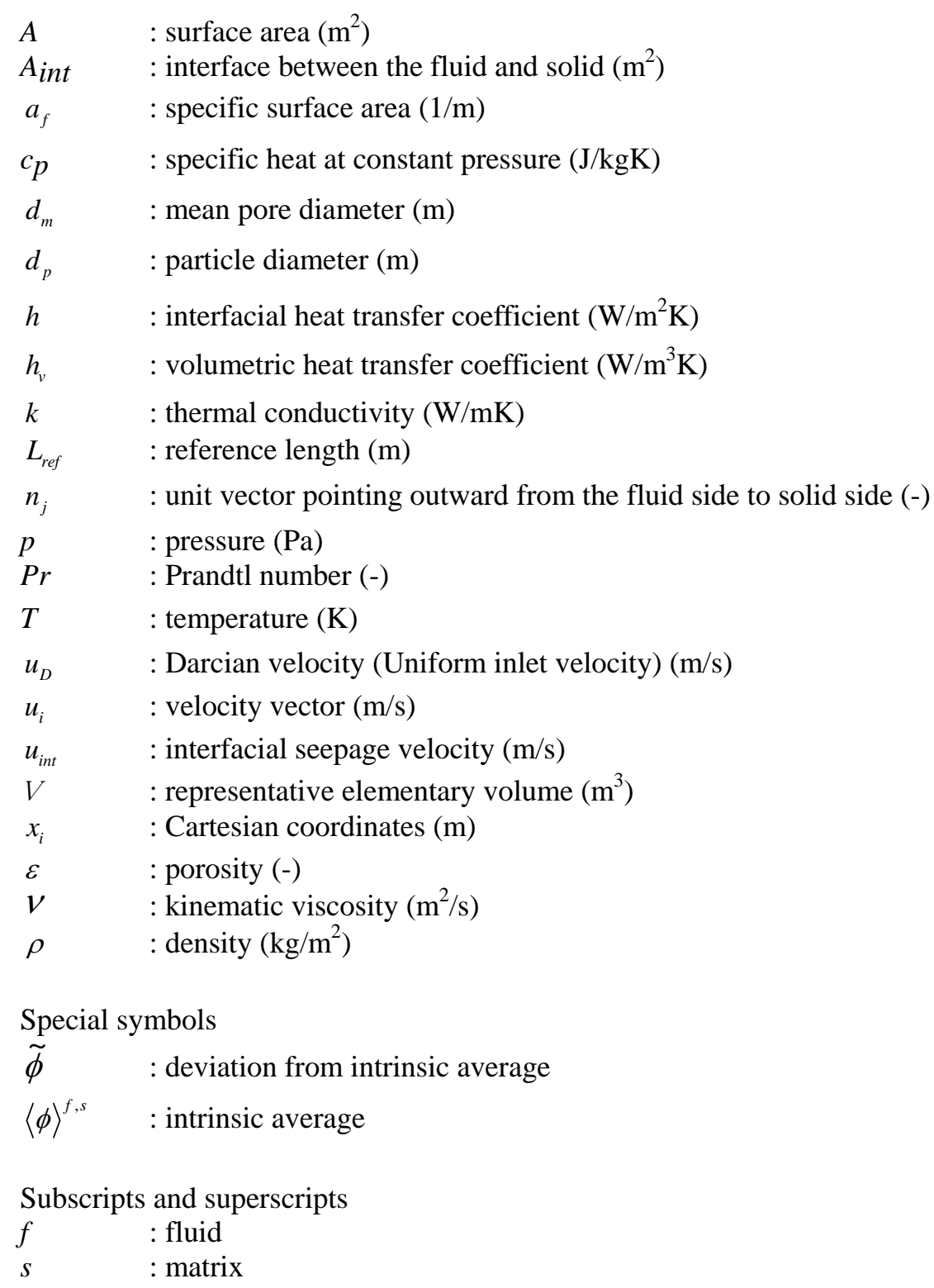

\section{REFERENCES}

[1] Wakao, N. and Kaguei, S., Heat and mass Transfer in packed beds, Gordon and Breach Science Pub., 1982.

[2] Kim, S. J. and Jang, S. P., Effects of the Darcy number, the Prandtl number and the Reynolds number on local thermal non-equilibrium, Int. J. Heat Mass Transfer, vol. 45, pp. 3885-3896, 2002.

[3] Polat, O., Crotogino, R. and Douglas, W., Through-drying of paper: A review, Advances in 
Drying, Majumdar, A. (Ed.), Vol. 5, pp. 263-299, 1992.

[4] Achenbach, E., Heat and flow characteristics in packed beds, Experimental Thermal and Fluid Science, vol. 10, pp. 17-27, 1995.

[5] Mahjoob, S. and Vafai, K., A synthesis of fluid and thermal transport models for metal foam heat exchangers, Int. J. Heat Mass transfer, vol. 51, pp. 3701-3711, 2008.

[6] Kim, S. J. and Hyun, J. M., A porous medium approach for the thermal analysis of heat transfer devices, Transport Phenomena in Porous Media III, Ingham, D. B. and Pop, I. (Ed.), pp. 120-146, 2005.

[7] Fu, X., Viskanta, R. and Gore, J. P., Measurement and correlation of volumetric heat transfer coefficients of cellular ceramics, Experimental Thermal and Fluid Science, vol. 17, pp. 285-293, 1998.

[8] Maiorov, V., Vasiliev, L. and Polyaev, V. M., Porous heat exchangers; Classification, application, J. Engineering Physics, vol. 47, pp. 1110-1123, 1984.

[9] Kar, K. K. and Dybbs, A., Internal heat transfer coefficient of porous metals, ASME Proc. Winter Annual Meeting, Phenix, Arizona, pp. 81-91, 1982.

[10] Kamiuto, K. and Yee, S. S., Heat transfer correlations for open-cellular porous materials, Int. Comm. Heat Mass Transfer, vol. 32, pp. 947-953, 2005.

[11] Younis, L. B. and Viskanta, R, Experimental determination of the volumetric heat transfer coefficient between stream of air and ceramic foam, Int. J. Heat Mass Transfer, vol. 36, pp. 1425-1434, 1993.

[12] Ichimiya, K., A new method for evaluation of heat transfer between solid material and fluid in a porous medium, ASME J. Heat Transfer, vol. 121, pp. 978-983, 1999.

[13] Liu, J. J., Sano, Y. and Nakayama, A., A mathematical model for determining the equivalent permeability in fractured porous media, Int. Comm. Heat Mass Transfer, vol. 36, pp. 220-224, 2009.

[14] Nakayama, A., PC-aided numerical heat transfer and convective flow, pp. 49-50, 103-115, CRC Press, Boca, Raton, 1995.

[15] Cheng, P., Heat transfer in geothermal systems, Advances in Heat Transfer, vol. 14, pp. 1-105, 1978.

[16] Quintard, M. and Whitaker, S., One and two equation models for transient diffusion processes in two-phase systems. Advances in Heat Transfer, vol. 23, pp. 369-465, 1993. 


\section{FIGURE CAPTIONS}

Figure 1 Unconsolidated porous medium

Figure 2 Comparison of the present formula with the empirical correlation for packed beds

Figure 3 Consolidated porous medium

Figure 4 Simplified model for a consolidated porous medium

Figure 5 Net heat transfer due to seepage flow

Figure 6 Comparison of the present expression with the experimental data of Fu et al.

Figure 7 Comparison of various correlations with the experimental data of Younis and Viskanta 